\title{
Designing Bioactive Delivery Systems for Tissue Regeneration
}

\author{
Hillary E. Davis ${ }^{1,2}$ and J. Kent Leach ${ }^{1}$ \\ ${ }^{1}$ Department of Biomedical Engineering, University of California, Davis, 451 Health Sciences Drive, 2303 Genome and \\ Biomedical Sciences Facility, Davis, CA 95616, USA; and ${ }^{2}$ School of Medicine, University of California, Davis, Sacramento, \\ CA 95817, USA
}

(Received 8 June 2010; accepted 20 July 2010; published online 30 July 2010)

Associate Editor Scott I. Simon oversaw the review of this article.

\begin{abstract}
The direct infusion of macromolecules into defect sites generally does not impart adequate physiological responses. Without the protection of delivery systems, inductive molecules may likely redistribute away from their desired locale and are vulnerable to degradation. In order to achieve efficacy, large doses supplied at interval time periods are necessary, often at great expense and ensuing detrimental side effects. The selection of a delivery system plays an important role in the rate of re-growth and functionality of regenerating tissue: not only do the release kinetics of inductive molecules and their consequent bioactivities need to be considered, but also how the delivery system interacts and integrates with its surrounding host environment. In the current review, we describe the means of release of macromolecules from hydrogels, polymeric microspheres, and porous scaffolds along with the selection and utilization of bioactive delivery systems in a variety of tissue-engineering strategies.
\end{abstract}

Keywords-Recombinant proteins, Growth factors, Delivery vehicles, Diffusion, Hydrogels, Microspheres, Scaffolds, Tissue engineering.

\section{INTRODUCTION}

Strategies to promote the repair or regeneration of diseased or absent tissue require an integrated approach that provides the necessary multiple cues for cells and tissues to develop adequate functionality. The sustained presentation of bioactive macromolecules such as growth factors and cytokines can stimulate host cells to migrate, proliferate, and differentiate toward a desired cell phenotype. ${ }^{11}$ Most proteins and nucleotide sequences have short half-lives, and thus

Address correspondence to J. Kent Leach, Department of Biomedical Engineering, University of California, Davis, 451 Health Sciences Drive, 2303 Genome and Biomedical Sciences Facility, Davis, CA 95616, USA. Electronic mail: jkleach@ucdavis.edu need to be administered periodically if given as bolus solution. ${ }^{6}$ Moreover, the systemic administration of bioactive factors is costly, can induce an immunogenic or other undesirable response by stimulating cells in a distant site, and neglects the spatial organization of drug gradients that are required by many tissues for maximum regenerative potential. To address the limitations of systemic delivery, biomacromolecules can be associated with drug delivery devices, which enable predictable control of the kinetics of therapeutic agent release. Within this review, we highlight the predominant mechanisms of drug delivery in current devices and provide recent examples of delivery strategies in use for tissue regeneration.

\section{DRUG DIFFUSION}

\section{Free Aqueous Diffusion}

Diffusion governs the release of macromolecules from the majority of controlled release systems. This physical phenomenon refers to the random walk of molecules resulting from thermal energy released from molecular collisions. Despite the arbitrariness of the molecular motion, a net transport of molecules from a region of higher concentration to one of lower concentrations is observed macroscopically. Additionally, transport ensuing from the bulk motion of fluids within the release medium, or convection, can be of importance at Péclet numbers (dimensionless group representing the ratio of convective to diffusive transport) greater than unity. At these instances where system length is greater than diffusion length, additional kinetics will need to be considered. However, this transport mechanism is beyond the scope of this review and can be found described in detail elsewhere. ${ }^{12,20,25}$ 
Diffusion of solutes in an aqueous solution can be described by the Stokes-Einstein equation. This correlation often specifies macromolecule size in terms of the hydrodynamic radius as an alternative to the hypothetical hard sphere used in the initial assumptions of this equation. In practice, macromolecules swell, solubilize, and demonstrate fluctuating conformations. ${ }^{35}$ Thus, the hydrodynamic radius accurately refers to the apparent size of the dynamic, hydrated molecule. ${ }^{74}$ Equation (1) calculates the free bulk diffusion coefficient as a function of the Boltzmann constant $\left(k_{\mathrm{B}}\right)$, absolute temperature $(T)$, solvent dynamic viscosity $(\mu)$, and macromolecule hydrodynamic radius $\left(r_{\mathrm{H}}\right)$.

$$
D=\left(k_{\mathrm{B}} T\right) /\left(6 \pi \mu r_{\mathrm{H}}\right)
$$

This approach is not adequate for all proteins, specifically proteins that significantly differ from a spherical geometry. For particles such as rigid rods and ellipsoids of revolution, equations relating hydrodynamic radii to the actual molecular dimensions are described by alternative relations. . $^{13,26,59,63}$

The free diffusion coefficient is predominantly dependent upon solvent temperature, viscosity, and solute size for particles diffusing in open solution. It is ideal to directly measure the diffusion coefficient at the same environmental conditions at which the majority of the data is obtained. As an alternative to experimental data, the Stokes-Einstein equation can be used to relate unknown coefficients assessed at different temperatures to known coefficients using Eq. (2).

$$
D_{2}=\left(T_{2} \mu_{1} D_{1}\right) /\left(T_{1} \mu_{2}\right)
$$

Table 1 lists free diffusion coefficients for several proteins used as either models or inductive agents in biomacromolecule delivery systems.

TABLE 1. Literature values of diffusion coefficients in water

\begin{tabular}{|c|c|c|c|}
\hline Protein & $\begin{array}{c}D_{\mathrm{w}}^{\mathrm{a}} \\
\left(\times 10^{-6} \mathrm{~cm}^{2} / \mathrm{s}\right)\end{array}$ & $\begin{array}{l}r_{\mathrm{H}} \mathrm{b} \\
(\mathrm{nm})\end{array}$ & $\begin{array}{c}\mathrm{MW} \\
\left(\times 10^{3}\right)\end{array}$ \\
\hline Bovine chymotrypsinogen ${ }^{72}$ & 0.71 & 3.5 & 24.0 \\
\hline $\mathrm{BSA}^{97}$ & 0.72 & 3.4 & 66.5 \\
\hline Bovine hemoglobin ${ }^{97}$ & 0.84 & 2.9 & 68.0 \\
\hline $\begin{array}{l}\text { Brain-derived neurotrophic } \\
\text { factor }^{89}\end{array}$ & 0.37 & 6.6 & 27.3 \\
\hline Epidermal growth factor ${ }^{93}$ & 1.3 & 1.9 & 6.60 \\
\hline Human immunoglobulin $\mathrm{G}^{65}$ & 0.38 & 6.5 & 146 \\
\hline Human immunoglobulin $\mathrm{M}^{65}$ & 0.18 & 14 & 970 \\
\hline $\mathrm{NGF}^{90}$ & 1.0 & 2.4 & 130 \\
\hline Porcine insulin ${ }^{97}$ & 1.3 & 1.8 & 5.80 \\
\hline VEGF $165^{17}$ & 0.52 & 4.7 & 19.0 \\
\hline
\end{tabular}
at $25^{\circ} \mathrm{C}$.

aValues adjusted for temperature using Eq. (2).

${ }^{\mathrm{b}}$ Calculated using Eq. (1).

\section{Diffusion Controlled Delivery Systems: Hydrogels}

Materials for tissue regeneration can confer additional structural hindrances to diffusing solutes, and in turn, notably affect the rate of diffusive transport. Vehicles for biomacromolecule delivery in tissue engineering often take the physical forms of hydrogels, microspheres, and porous scaffolds. Hydrogels are polymeric networks that imbibe large amounts of water while remaining insoluble. Simple design parameters such as molecular weight of the polymer, crosslinking density, polymer-protein interactions, and gel degradation rate can control the hydrogel structure which, in turn, can affect diffusion coefficients. ${ }^{49}$ Diffusion-controlled hydrogel delivery systems can be classified as either reservoir or matrix systems (Fig. 1). In a reservoir system, a core of biomacromolecules is surrounded by an inert polymeric membrane. The drug diffuses through the membrane at a controllable rate. If the concentration of biomacromolecules within the membrane is static, then the concentration gradient throughout the membrane will be constant and the reservoir system will provide a steady release rate of solute. This occurs when the initial solution encapsulated in the membrane is saturated, leading to zeroorder release kinetics. ${ }^{69}$ The release of solutes from such a reservoir system is governed by Fick's first law of diffusion

$$
J=-D \nabla C
$$

where $J$ is the flux of the solute, $D$ the diffusion coefficient of the solute in the membrane, and $\nabla C$ the spatial gradient of solute concentration. To simplify modeling, it is generally assumed that the drug diffusion coefficient is constant in reservoir systems. ${ }^{49}$ As mentioned above, the magnitude of diffusive flux is driven by the concentration gradient across the membrane. Macromolecule release rate can be determined by calculating the product of the flux and the surface area. For example, the release rate for cylindrical reservoir systems is represented by

$$
d M / d t=(2 \pi L D K \Delta C) / \ln \left(r_{\mathrm{o}} / r_{\mathrm{i}}\right)
$$

where $d M / d t$ is the release rate, $L$ the length of the cylinder, $D$ the diffusion coefficient, $K$ the partition coefficient between membrane and core, $\Delta C$ the difference in concentration between the inside and outside of the membrane, $r_{\mathrm{o}}$ and $r_{\mathrm{i}}$ are the outer and inner radius of the membrane, respectively. ${ }^{50,96}$

For such membrane reservoir systems, release kinetics are independent of molecular size or weight of the active molecule. This is a great advantage, as the same membrane scheme can be used for similar biomacromolecules without repeating release characterization measurements. However, reservoir systems are 

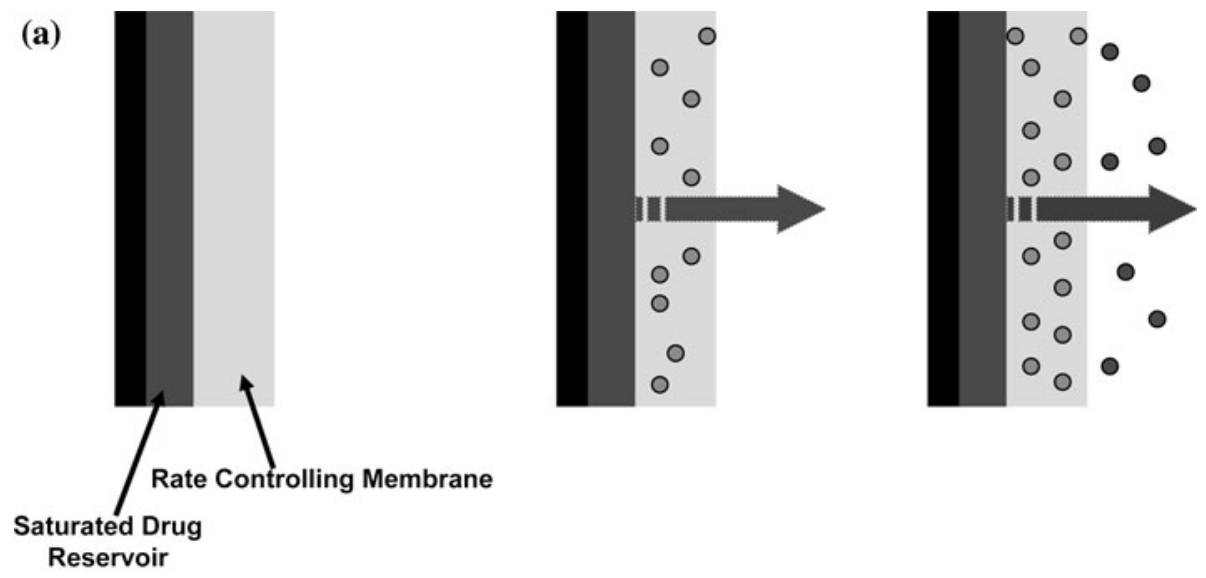

(b)
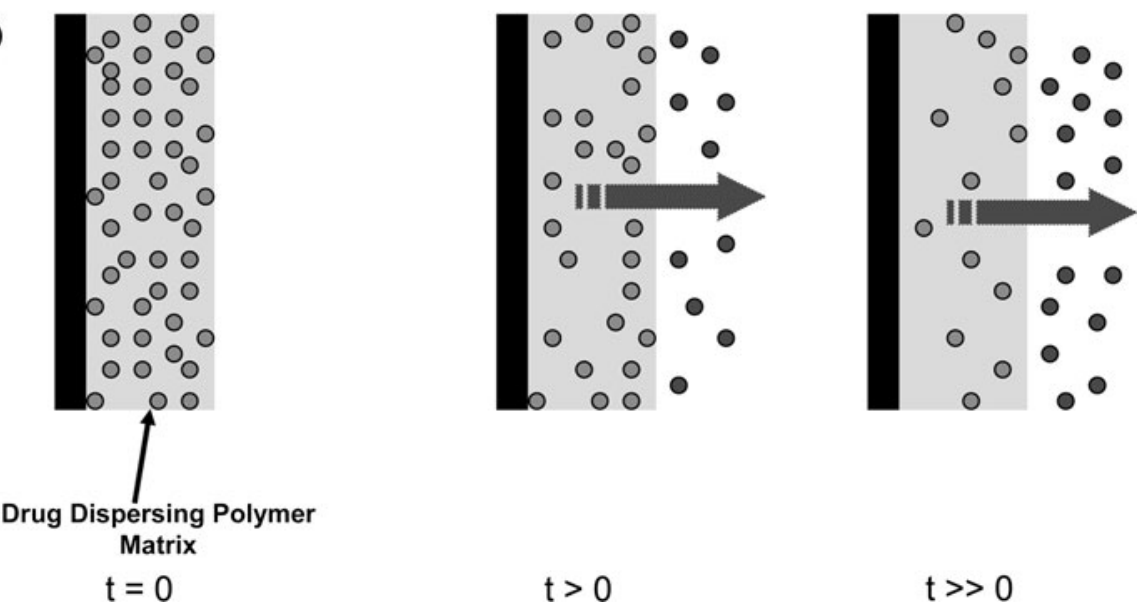

$t>0$

$t>0$

FIGURE 1. The sustained release of macromolecules is achieved by incorporation in reservoir or matrix systems. (a) Delivery from a reservoir system can be tailored by adjusting the properties of the rate-controlling membrane including thickness and porosity. (b) Delivery from a matrix system representing uniform initial distribution throughout the polymer phase.

often susceptible to bulk release of their contents if membrane flaws are present. Hence, stringent manufacturing procedures are necessary to ensure appropriate drug diffusion.

In a matrix system, the biomacromolecule is initially distributed homogeneously throughout the construct's volume. If a constant diffusion coefficient $(D)$ is assumed, one-dimensional diffusion can be modeled using Fick's second law of diffusion ${ }^{61}$

$$
d C / d t=D\left(d^{2} C / d x^{2}\right)
$$

Several important assumptions are associated with Eq. (5) including (1) the hydrogel is highly swollen such that the solutes are significantly smaller than the thickness of the system; (2) the diffusivity of the solute is constant throughout the construct's volume; (3) degradation of the matrix is negligible; (4) perfect sink conditions are preserved; and (5) absence of solutecarrier interactions. ${ }^{82}$ If the initial drug concentration in the matrix is much higher than its solubility, steadystate kinetics can be assumed for the initial portion of the release curve. Diffusion coefficients can be determined experimentally (Table 2) and, with the appropriate boundary conditions, Eq. (5) can be solved. For the simplest geometry of a matrix system, a slab with a thickness, $L$, the following boundary conditions apply

$$
\begin{array}{lll}
t=0 & -L / 2<x<L / 2 & C=C_{1} \\
t>0 & x \pm L / 2 & C=C_{0}
\end{array}
$$

where $C_{1}$ is the initial uniform drug concentration and $C_{0}$ is the concentration of drug present in the bulk solution outside of the construct. ${ }^{71}$ Solving Fick's second law of diffusion using the separation of variables technique leads to the analytical solution

$$
\begin{aligned}
M_{t} / M_{\infty}= & 1-\sum_{n=1}^{\infty}\left(8 /(2 n+1)^{2} \pi^{2}\right) \\
& \times \exp \left[-(2 n+1)^{2} \pi^{2} D t / L^{2}\right]
\end{aligned}
$$

where $M_{t}$ is the mass of biomacromolecule released at time $t$ and $M_{\infty}$ is the final amount of molecule released which approaches $C_{1}$ as time goes to infinity. 
TABLE 2. Selected literature values of effective diffusion coefficients of proteins through hydrogels.

\begin{tabular}{lcl}
\hline Protein & $D_{\mathrm{e}}\left(\times 10^{-6} \mathrm{~cm}^{2} / \mathrm{s}\right)$ & \multicolumn{1}{c}{ System } \\
\hline BSA & 0.46 & Low molecular weight calcium-alginate gel at $25^{\circ} \mathrm{C}^{2}$ \\
BSA & 0.45 & Glycidyl methacrylate modified hyaluronic acid gel at $37^{\circ} \mathrm{C}^{47}$ \\
BSA & 0.014 & Poly(ethylene glycol)-diacrylate gel at $25^{\circ} \mathrm{C}^{62}$ \\
BMP-2 & $2.9 \mathrm{E}-4$ & Poly(ethyl methacrylate)/tetrahydrofurfuryl methacrylate gel at $37^{\circ} \mathrm{C}^{58}$ \\
$\begin{array}{l}\text { Insulin-like growth } \\
\text { factor I }\end{array}$ & 1.59 & Fibrin gel at $25^{\circ} \mathrm{C}^{55}$ \\
$\begin{array}{l}\text { Myoglobin } \\
\text { NGF }\end{array}$ & 0.064 & Poly(ethylene glycol) diacrylate gel at $37^{\circ} \mathrm{C}^{24}$ \\
\hline
\end{tabular}

Equation (6) can be recast in a more familiar form as an error function series ${ }^{5,22,23}$

$$
\begin{aligned}
M_{t} / M_{\infty}= & 4\left(D t / L^{2}\right)^{0.5} \\
& \times\left\lfloor\pi^{-0.5}+2 \sum_{n=1}^{\infty}(-1)^{n} \operatorname{ierfc}\left(n L /\left(2(D t)^{0.5}\right)\right)\right\rfloor
\end{aligned}
$$

For short times, the equation is reduced to the first term in the brackets and Eq. (7) becomes

$$
M_{t} / M_{\infty}=4\left(D t /\left(\pi L^{2}\right)\right)^{0.5}
$$

It is important to note that this approximation is only valid for the first $60 \%$ of drug released. ${ }^{71}$ Thus, at short times, the fraction of protein released is directly proportional to some constant multiplied by the square root of time. If biomacromolecule delivery for a simple slab matrix system was independent of time, zero-order release kinetics would be observed.

Most matrix systems can be characterized by both Fickian and non-Fickian diffusion schemes. A generalization of these transport mechanisms is given by the power law, first applied to matrix systems by Peppas ${ }^{60}$

$$
M_{t} / M_{\infty}=k \times t^{n}
$$

where $k$ is a constant that is determined by the structural and geometrical characteristics of the release device and $n$ is the 'release exponent,' which represents the joint contributions of both Fickian and zero-order diffusion mechanisms involved in protein release. For slabs, the system is purely diffusion-controlled for $n=0.5$, whereas for $n=1$, the release mechanism is governed by zero-order kinetics. Due to the initial assumptions present in Eq. (9), the power law is only valid for the initial $60 \%$ of the release profile. Additionally, $k$ and $n$ are not independent of geometry and thus cylindrical and spherical systems will have different interpretations of these variables. ${ }^{82}$ However, Eq. (9) represents a simple and comprehensive method of describing the general biomacromolecule release profile from matrix systems.

Although diffusion-controlled release kinetics are the most widely applicable to drug delivery from matrix systems, there are numerous other mechanisms of biomacromolecule release from hydrogels present in the literature. Swelling-controlled, chemically controlled, temperature-, pH-, magnetic-, and electricsensitive hydrogel release systems have all been extensively studied. Several thorough reviews are available on these subjects. ${ }^{4,6,49}$

\section{Diffusion-Controlled Delivery Systems: Microspheres}

Polymeric microspheres are a common type of controlled-release device and have received considerable interest from the pharmaceutical industry as a vehicle for optimizing local drug concentrations and increasing patient compliance. ${ }^{21}$ Microspheres composed of poly(lactide-co-glycolide) (PLG) are commonly used due to their lack of toxicity, biocompatibility, and biodegradability. ${ }^{8}$ PLG microspheres are capable of entrapping both hydrophobic and hydrophilic biomacromolecules as determined by fabrication procedure: an oil-in-water $(\mathrm{o} / \mathrm{w})$ emulsion/ solvent evaporation technique encapsulates hydrophobic materials while hydrophilic solutes can be entrapped by the water-oil-in-water $(\mathrm{w} / \mathrm{o} / \mathrm{w})$ technique. ${ }^{41,100}$ The properties of synthetic polymer microspheres including polymer molecular weight and composition are highly tailorable, thus enabling the tight regulation and control of protein delivery. ${ }^{57}$ Although the importance of PLG-based controlled release biomacromolecule systems is steadily increasing, as evidenced by the number of PLG-based microparticles available on the market, the transport mechanisms of drug release have yet to be fully elucidated. ${ }^{45}$

Recent studies have suggested that the dominant mechanism for protein release through a homogeneous polymer phase is due to diffusion of the drug. Mathematical models using solutions to Fick's second law for a spherical geometry correlate well to experimentally obtained release profiles. ${ }^{32,45}$ However, a purely diffusion-based model fails to account for the initial burst release of proteins. As an attempt to reconcile this difference along with obtaining an even more robust and versatile predictor of molecule release, 
various models such as the Higuchi equation were formed that accounted for diffusion, polymer degradation, and microsphere size distribution. ${ }^{8,66}$ Although these models yielded a more precise fit to experimental data, they failed to adequately relate the initial burst release of proteins to a transport mechanism.

\section{Diffusion-Controlled Delivery Systems: Porous Scaffolds}

The accurate characterization and modeling of protein release from and within porous scaffolds is complex, as there are many variables that can affect diffusion including polymer degradation, matrix crystallinity, porosity, geometry, or other mass transport or chemical phenomena. ${ }^{81}$ When the concentration of drug molecules within a porous matrix exceeds drug solubility in the aqueous solution, a moving boundary between dissolved and dispersed drug may arise. A pseudo-steady-state model of Higuchi has been modified to describe these systems ${ }^{77}$

$$
M_{t}=A \sqrt{D_{\text {eff }} C_{\mathrm{S}}\left(2 C_{\mathrm{A}}-\varepsilon C_{\mathrm{S}}\right) t}
$$

where $D_{\text {eff }}$ is the effective diffusion coefficient, $A$ the surface area for release, $C_{\mathrm{S}}$ the solubility of the macromolecule in the aqueous medium, $C_{\mathrm{A}}$ the initial amount of molecule present per unit volume of the matrix, $\varepsilon$ the scaffold porosity, and $t$ is time. Saltzman and Langer ${ }^{78}$ used percolation theory to describe effective diffusion coefficients for protein transport through non-biodegradable, porous matrices in the context of Fick's second law. For biodegradable systems where porosity increases with time, Monte Carlo methods have been employed to achieve a more accurate, stochastic description of biomacromolecule diffusion. ${ }^{80}$ Recently, a model has been proposed that describes both surface and bulk erosion as a means to determine effective diffusion coefficients. ${ }^{73}$ Although no model has been developed that accounts for all of the important processes involved in diffusion from porous scaffolds, these models save time and effort when characterizing biomacromolecule release from porous systems.

\section{CONTROLLED DRUG DELIVERY SYSTEM STRATEGIES}

\section{Particulate Systems}

Particulate systems, which include microparticles $(1-1000 \mu \mathrm{m})$ and nanoparticles $(1-1000 \mathrm{~nm})$, do not require major surgical procedures for implantation. ${ }^{84}$ These systems have been developed as an alternative to scaffolds and hydrogels to deliver biomacromolecules in a minimally invasive manner. Advantages of these systems are numerous: they can be evenly dispersed into a target tissue avoiding drug gradients, size distribution of the particles is easily controlled, the particles may have pores allowing for variable release kinetics, and each particle has a high mass ratio of biomacromolecule to polymer. ${ }^{34,86}$ However, particulate systems are not desirable for all tissue engineering applications, as the particles will not remain in the proximal locales for tissues subjected to high fluid shear stresses. Still, particulate systems have found numerous applications in tissue-engineering drug delivery schemes. ${ }^{7,39,79,99}$

Particulate systems can be prepared from naturally occurring polymers such as cellulose, alginate, chitin, and chitosan or with synthetic polymers such as PLA and PLG. ${ }^{9,28,36}$ Alginate beads were among the first particulate systems and can be fabricated by extruding alginate solutions into a solvent containing divalent cations, with calcium being the most widespread. ${ }^{51}$ However, Jay and Saltzman ${ }^{40}$ recently demonstrated that varying the type and concentration of ionic crosslinker allowed for additional control over VEGF release from small alginate microparticles. Using spherical release assumptions, they fit VEGF release profiles obtained by ELISA to Eq. (9) and demonstrated that release from $700 \mathrm{mM} \mathrm{CaCl}$-crosslinked particles was anomalous (non-Fickian diffusion), whereas release from $700 \mathrm{mM} \mathrm{ZnCl} \mathrm{Zn}_{2}$-crosslinked particles was governed by super case II transport (polymeric erosion). The conventional model for diffusion of macromolecules through $\mathrm{Ca}$-alginate beads is the spherical variant of Eq. (3), which accurately predicts release rates for small proteins not severely influenced by charge and $\mathrm{pH}$ of the alginate matrix, such as insulin. ${ }^{67}$ Larger proteins require the addition of convective terms to Eq. (3) to reflect initial bead shrinkage during curing and subsequent swelling once placed in a physiological-buffered solution in order to avoid under-estimating diffusion coefficients. Thus, alginate beads may be ideal for dual delivery systems, as two proteins of varying size will demonstrate vastly different release kinetics. Alginate's relatively mild gelation process has enabled both proteins and cells to be incorporated into particles with retention of full biological activity. Moreover, pore size and degradation rate can be controlled by varying the purity and type of alginate and surface coating agent. ${ }^{44,68}$ Elcin et al. ${ }^{29}$ delivered vascular endothelial growth factor (VEGF) via alginate microspheres to a rat subcutaneously. The defect site had increased neovascularization over the control at 3 weeks, demonstrating that biological activity of the growth factor was preserved.

An especially exciting application for PLG microspheres may be for the controlled delivery of growth factors in the central nervous system to combat 
neurodegenerative diseases such as Alzheimer's or Parkinson's using direct brain implantation. ${ }^{30,104}$ Recently, PLG microparticles were functionalized by incorporating palmitic acid on the surface to facilitate the attachment of biotinylated ligands. ${ }^{31}$ Thus, targeting ligands such as antibodies can potentially be attached to microparticles and direct growth factors to diseased brain tissue sites.

\section{Hydrogel Matrices}

The simplest technique to add proteins to hydrogel systems is through direct loading into the polymeric matrices (Fig. 2a). Since protein release is generally diffusion-controlled through aqueous pores within the hydrogel, these systems only allow for drug release over short periods of time. Typical release profiles demonstrate a rapid burst while the hydrogel imbibes water, followed by the continued discharge of the remaining protein as the gel network relaxes. ${ }^{38,52}$ Varying cross-linking densities can easily lead to modified release profiles. ${ }^{49}$ Other methods of tailoring release kinetics in direct loading systems include altering the hydrogel microenvironment by controlling factors such as temperature, $\mathrm{pH}$, or ionic strength. For example, Yamamoto et al. ${ }^{102}$ demonstrated that gelatin hydrogels with a lower isoelectric point better retained basic fibroblast growth factor (bFGF) and transforming growth factor- $\beta 1$ (TGF- $\beta 1$ ) compared to basic gelatin hydrogels.

An interesting variation on direct loading of proteins within a hydrogel is to deliver cells and multiple (a)

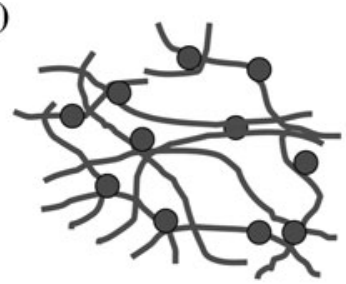

(c)

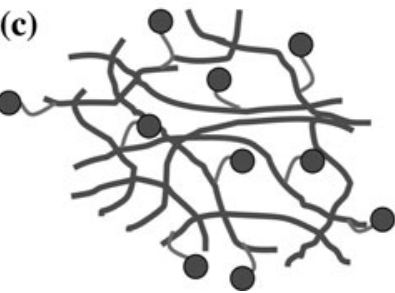

(b)

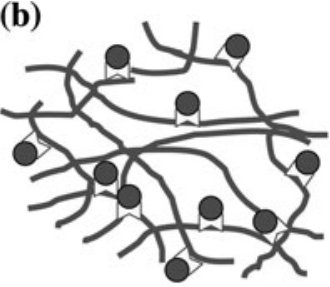

(d)

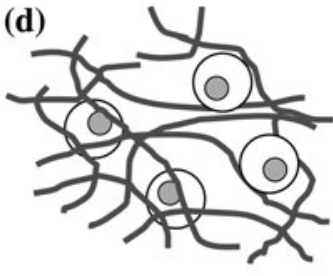

FIGURE 2. Schematic representation of common methods of macromolecule incorporation into hydrogel systems for controlled release and presentation. (a) Direct loading. (b) Immobilization through bonding to the polymer backbone. (c) Tethering of proteins to the polymer chains by linking with bi-domain peptides. (d) Encapsulation of macromolecules within particulate systems for subsequent mixture within the hydrogel. growth factors concurrently. Simmons et al. ${ }^{85}$ loaded growth factors for bone generation directly into RGDmodified alginate matrices at physiological concentrations along with rat bone marrow stromal cells. When a single growth factor was delivered alone, either bone morphogenetic protein-2 (BMP-2) or transforming growth factor- $\beta 3$ (TGF- $\beta 3$ ), little bone formation was observed. Such results were not unusual, as prior studies demonstrated that bone regeneration with delivery of a single growth factor required supraphysiological protein concentrations. ${ }^{75}$ However, the simultaneous delivery of BMP- 2 and TGF- $\beta 3$ resulted in enhanced bone formation. Perhaps less growth factor was necessary since the diffusion path length to bone progenitor cells was shorter, or delivering multiple growth factors has synergistic effects on cell differentiation. Altogether, this study describes a powerful tool for overcoming the shortcomings of directly loaded gels and can be employed in other polymeric delivery devices to improve cell response.

The early release burst effect can be mitigated by fabricating hydrogels with biomacromolecules bonded to the polymeric architecture (Fig. 2b). Andrades et al. ${ }^{3}$ produced recombinant fusion proteins of TGF$\beta 1$ that possessed an auxiliary von Willebrand factor-derived collagen binding domain that allowed noncovalent immobilization within collagen type I-based materials. Compared to native TGF- $\beta 1$, the incorporation of this fusion protein resulted in higher levels of migration, growth, and differentiation of murine bone marrow mesenchymal cells in collagen gels. In a similar approach, Sakiyama and Hubbel1 ${ }^{76}$ developed a fibrinbased heparin-containing delivery system to modulate the release of beta-nerve growth factor ( $\beta$-NGF). Heparin binding sites were covalently bonded to the fibrin hydrogel using the transglutaminase activity of factor XIIIa resulting in the immobilization of heparin. The growth factor bound non-covalently to the heparin, allowing for the slow diffusion-based release of $\beta$-NGF. These systems showed enhanced neurite extension over free neurotrophins (NTs) directly loaded into fibrin matrices. However, since this study was conducted in vitro, the biological activity of the immobilized proteins in the physiological environment remains undefined. Enzymatic cleavage of both heparin and growth factors may result in varying release and inactivation rates. Tethering of growth factors to the polymer backbone (Fig. 2c) has been successfully employed for other macromolecules including BMP-2, VEGF, TGF- $\beta 1$, and platelet-derived growth factor (PDGF). $1,33,64$

For long-term applications, it may be necessary to maintain control over protein activity and stability in order to maximize their inductive potential and resulting regenerative benefits. To achieve these goals, 
separate release systems can be incorporated into hydrogels (Figs. 2d and 3). Often these secondary release systems take the form of micro- or nanoparticles and can be directly loaded into the pre-gelled solution. This strategy also enables the sustained delivery of multiple proteins with distinct temporal sequences. Burdick et al. ${ }^{14}$ encapsulated NT-3 in degradable microspheres of PLGA which were then directly loaded into the poly(ethylene glycol) (PEG) hydrogel phase containing ciliary-neurotrophic factor (CNTF). Release profiles demonstrated rapid release of CNTF in the hydrogel phase and a slower, continuous release of NT-3 from the degradable microspheres. It is easily envisioned that these release kinetics can be tailored by altering the polymer chemistry, molecular weight, and size of the microspheres or the cross-linking density or molecular weight of the hydrogel. Healthy tissue formation is regulated by multiple soluble cues that are presented over varying temporal durations. By employing further hydrogel modification techniques, such as covalently bonding factors to the hydrogel polymer, more signals can be delivered at different time sequences. These composite systems possess the potential to more
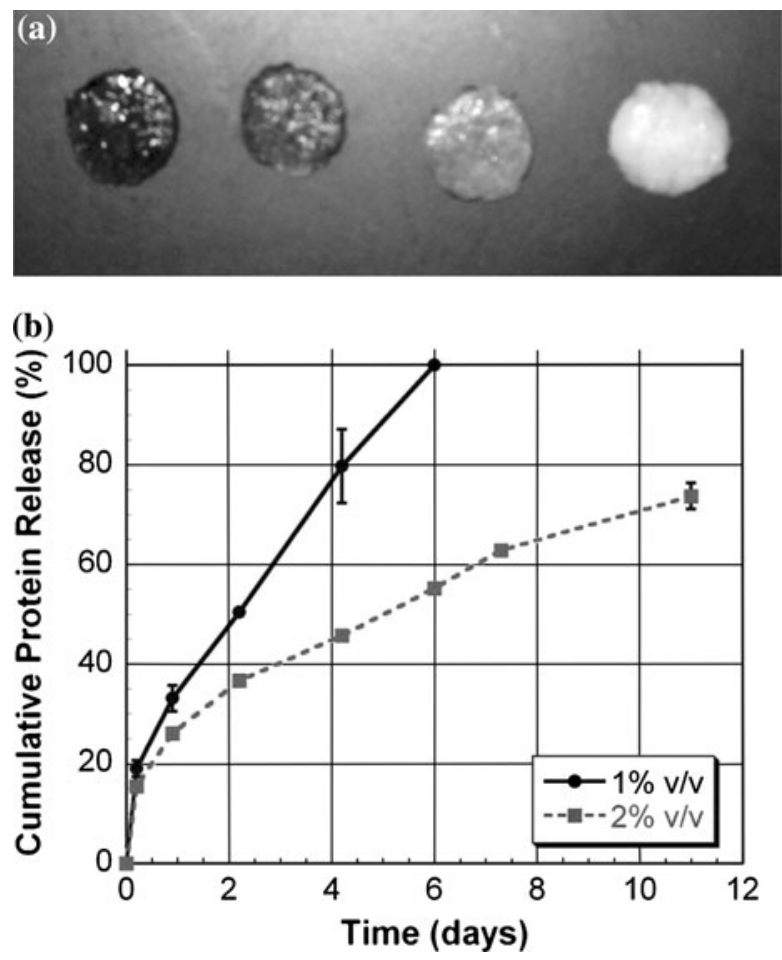

FIGURE 3. Protein release can be modulated by fabricating particulate/hydrogel composite systems or varying the hydrogel composition. (a) Alginate gels containing $0,2.5,5$, and $10 \mathrm{mg}$ PLG microspheres $/ \mathrm{mL}$ of gel (from left to right). (b) The release of microsphere-encapsulated protein entrapped in alginate gels is controlled by varying the alginate concentration. accurately mimic the biological cues that occur naturally in vivo.

\section{Porous Polymer Scaffolds}

Formation of growth factor-loaded porous solid scaffolds can be attained through several approaches. The main strategies involve either one step direct formation of growth factor-loaded scaffolds or formation of microspheres with encapsulated protein which can then be compression molded and fused to form scaffolds. Degradation time and mechanical properties of these tissues should match the speed of regeneration for its appropriate tissue. Complete reviews of materials and scaffold processing techniques can be found elsewhere. ${ }^{46,87}$

Mooney et $a l .{ }^{53}$ were the first to produce porous polymeric matrices by means of saturating disks of PLG with carbon dioxide gas at high pressure. By combining this process with particulate leaching techniques, matrices with a well-controlled porosity and interconnectivity were fabricated, avoiding the concerns of using high temperatures and organic solvents that were necessary for conventional strategies for forming porous matrices. ${ }^{37}$ The improved fabrication conditions enabled the sustained release of inductive growth factors from these matrices. The potent angiogenic factor, VEGF, was incorporated into gasfoamed scaffolds using two schemes: (1) covalent association of the protein through lyophilization with the polymer prior to scaffold formation; and (2) encapsulating the growth factor in microparticles and then fabricating the scaffold from drug-loaded microspheres. Richardson et al. $^{70}$ capitalized on this increased functionality by delivering multiple angiogenic factors with distinct release profiles. PDGF was encapsulated within the microspheres, whereas VEGF was incorporated into the bulk phase of the scaffold. Figure 4 illustrates schematically the dual growth factor delivery system reported by Richardson et al. These dual-release scaffolds led to an enhanced amount of vessel formation and maturation in vivo. This study demonstrated that regulating local delivery of multiple growth factors led to a more fully developed capillary network. This concept has wide applicability to other inductive factors and tissues and represents a strategy that should be applied to future tissue-engineering technologies.

The formation of scaffolds using electrospinning techniques enables the encapsulation and embedding of proteins into fibers using coaxial and emulsion fabrication procedures. ${ }^{83}$ These matrices possess a high surface area, which allows for high drug loadings. Chew et al. ${ }^{19}$ mixed human NGF in a copolymer of $\varepsilon$-caprolactone and ethyl ethylene phosphate in 
dichloromethane, yielding a protein-polymer suspension prior to electrospinning. The resulting fibers demonstrated randomly distributed proteins throughout their volumes. After a moderate burst of approximately $20 \%$ of the total incorporated NGF, the protein was released in a controlled fashion via diffusion. Zhang et al. ${ }^{103}$ reported a dramatic reduction in the burst effect, coupled with an extended duration of sustained release, by loading proteins into the cores of coaxial fibers. Although the release kinetics were still primarily governed by diffusion as in the embedded systems, the core-loaded fibers behaved more similarly to reservoir devices. This technique also avoids extended protein exposure to harsh organic solvents necessary for blend-electrospinning processes, representing another advantage for this method. The utility of scaffolds fabricated with this technique is limited to certain tissues due to the mechanical properties of the resulting matrices. However, this process has emerged as a valuable tool to tailor protein release kinetics.

In addition to incorporating biomacromolecules directly into the structure of a scaffold, alternative methods have been used to gain further control over localized delivery. Surface coatings can be used to not only increase the incorporation efficiency of the protein at the scaffold's exterior, but also to encourage

(a)

$t=0$

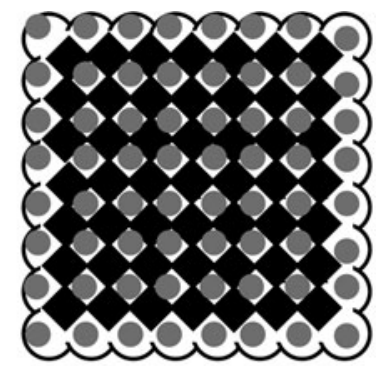

(c)

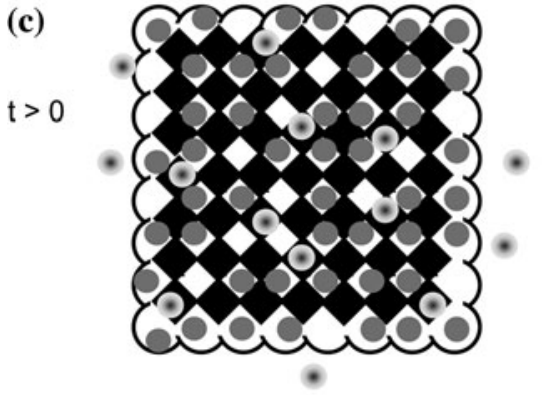

increased cell interaction with the scaffold. ${ }^{27,101}$ Collagen-coated scaffolds have been fabricated as a means for encouraging cell attachment as well as delivering growth factors rapidly after implantation (Fig. 5). ${ }^{48}$ However, the submersion of substrates into protein solutions yields only small amounts of adsorbed protein. As an alternative, biomacromolecules can be conjugated to the surface of a scaffold, hence prolonging the release kinetics. Jeon et al. ${ }^{42}$ covalently bound heparin to the surface of PLG scaffolds using carbodiimide chemistry which, in turn, enabled the linkage of BMP-2. These surface-functionalized scaffolds induced bone formation to a much greater degree than directly loaded BMP-2 scaffolds, likely due to factor retention at the cell surface and continued stimulation, suggesting surface tethering as another important strategy for tissue regeneration.

As an alternative to stiffer biodegradable polyesters, elastomers such as poly(glycerol-co-sebacate) and trimethylene carbonate have gained popularity because they can provide stability and structural integrity within a mechanically dynamic environment without irritation to host tissues while exhibiting mechanical properties similar to those of soft tissues. ${ }^{56,98}$ These biomaterials have potential applications in tissue engineering and drug delivery toward the (b)

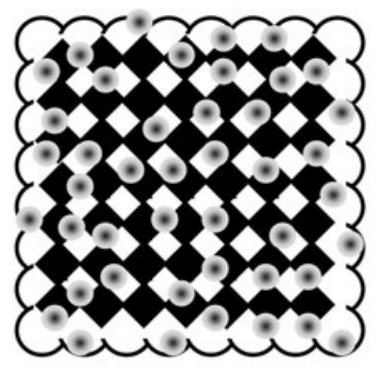

(d)

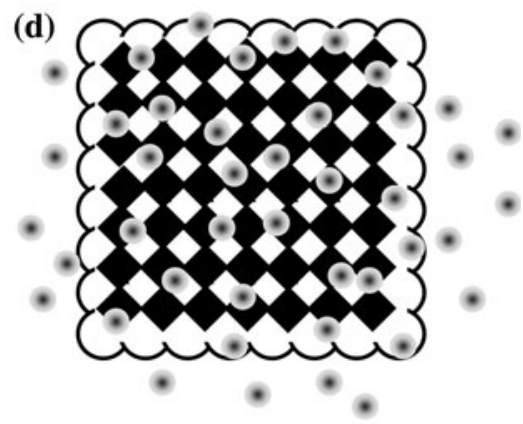

(O) Protein Encapsulated in PLG Microsphere

Protein
Pore

FIGURE 4. Biomacromolecule release kinetics can be controlled by the incorporation method and location within the substrate. (a) Growth factors and cytokines are encapsulated within polymeric microspheres and subsequently used to fabricate porous scaffolds. The protein is embedded more deeply within the biomaterial, and release is dependent upon polymer degradation and/or diffusion through the polymer layer and transport through the porous structure. (b) The covalent association of the protein with the biomaterial results in the macromolecule residing on or near the pores of the substrate and yields more rapid release. 

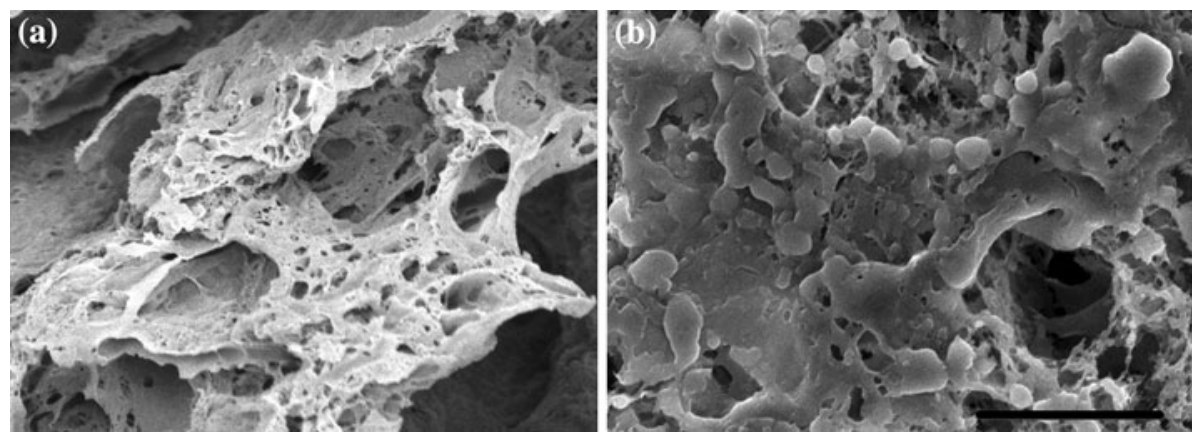

FIGURE 5. Biomacromolecule release rate can be tailored by depositing a coating on the delivery vehicle as observed with scanning electron microscopy. (a) Macroporous scaffold composed of PLG exhibits a range of pore sizes to enable drug release, cellular invasion, and nutrient transport. (b) Many smaller pores are occluded upon coating the scaffold with a collagen solution. $800 \times$ magnification; scale bar represents $50 \mu \mathrm{m}$.

development of small-diameter vascular grafts, ${ }^{54,88}$ cardiac patches, ${ }^{18}$ nerve conduits, ${ }^{91}$ and urological applications. ${ }^{94}$ Reservoir-based elastomeric devices were fabricated from poly(glycerol-co-sebacate) for sustained antibiotic release over 10 days. ${ }^{94}$ Orifices of varying diameters were drilled into the polymer using an excimer laser microablation technique to facilitate ciprofloxacin release. The release rate was dependent on orifice size, with orifice diameters of $100-150 \mu \mathrm{m}$ achieving zero-order release kinetics. Poly(trimethylene carbonate) was used to release two common inductive proteins, VEGF and hepatocyte growth factor, over 10 days. ${ }^{16}$ The highly bioactive proteins could be delivered simultaneously with similar, controlled release kinetics, or sequentially when entrapped separately within a dual-layered cylinder. The release kinetics were controlled by varying elastomer crosslinking density, achieved by modulating prepolymer molecular weight and resulting mechanical properties of the elastomer. Importantly, the great majority of protein is released from these surface-eroding materials prior to substantial mass loss and mechanical failure, potentially providing advantages to other materials such as PLGA for use in tissue repair.

\section{METHODS TO INCREASE PROTEIN STABILITY}

The production of biodegradable drug delivery devices containing stable, therapeutic proteins remains a major challenge. For example, encapsulation of factors in PLG devices using a double emulsion method leads to denaturing of secondary and tertiary structures due to protein-organic solvent and protein-polymer hydrophobic interactions. ${ }^{92}$ Additionally, when the polymer degrades over time, acidic degradation products result in further protein destabilization. ${ }^{43,95}$ Schwendeman and colleagues ${ }^{105}$ addressed this limitation by co-incorporating magnesium hydroxide; a strategy that maintained structure and function of encapsulated bovine serum albumin (BSA), bFGF, and BMP-2. For protein incorporation into hydrogels, bioactive factors risk denaturing while unprotected in the pre-gelled solution, and the addition of chemical initiators and free radicals can affect the biological activity of the proteins. Approaches to overcoming protein instability include chemically modifying the protein (e.g., PEGylation) or using additives such as sugars, salts, or other molecules to preferentially hydrate the native protein back to its native conformation. ${ }^{10}$ Although this technique is protein and polymer dependent, as varying protein isoelectric points will affect outcomes, it emphasizes a need for investigators to consider environmental variables before blindly adding proteins to polymer devices for delivery.

\section{OUTLOOK AND CONCLUSION}

Controlled delivery of biomacromolecules is emerging as an important component of the field of tissue engineering and regenerative medicine, as delivering matrices and cells alone may not be sufficient to recapitulate all aspects of healthy tissue. In particular, aspects of tissue formation (e.g., cell differentiation) that have been shown during normal development to depend on specifically timed delivery of soluble inductive factors may especially benefit from such controlled delivery approaches. Designing systems for macromolecule delivery is not a simple task, as different tissues require the presentation of various factors at specific times, thereby necessitating distinct release profiles. Although drug delivery devices now possess the potential to deliver multiple growth factors, additional research is merited to generate a sufficient understanding of the necessary temporal patterns of presentation to neighboring cells. Additionally, further studies must be conducted on delivery systems that can target intracellular compartments of tissues, as this would avoid biomacromolecule waste and increase the 
system efficacy. The development of new approaches to combat the "burst release" phenomenon is necessary, as well as techniques to bridge the disconnect between theoretical and experimental studies of drug delivery. Controlled biomacromolecule devices have significantly advanced the field of tissue engineering during their brief existence, and an increased understanding of the needs of each tissue will only enhance their impact.

\section{OPEN ACCESS}

This article is distributed under the terms of the Creative Commons Attribution Noncommercial License which permits any noncommercial use, distribution, and reproduction in any medium, provided the original author(s) and source are credited.

\section{REFERENCES}

${ }^{1}$ Aizawa, Y., N. Leipzig, T. Zahir, and M. Shoichet. The effect of immobilized platelet derived growth factor AA on neural stem/progenitor cell differentiation on celladhesive hydrogels. Biomaterials 29:4676-4683, 2008.

${ }^{2}$ Amsden, B. Solute diffusion in hydrogels. An examination of the retardation effect. Polym. Gels Networks 6:13-43, 1998

${ }^{3}$ Andrades, J. A., B. Han, J. Becerra, N. Sorgente, F. L. Hall, and M. E. Nimni. A recombinant human TGF-beta 1 fusion protein with collagen-binding domain promotes migration, growth, and differentiation of bone marrow mesenchymal cells. Exp. Cell Res. 250:485-498, 1999.

${ }^{4}$ Bajpai, A. K., S. K. Shukla, S. Bhanu, and S. Kankane. Responsive polymers in controlled drug delivery. Prog. Polym. Sci. 33:1088-1118, 2008.

${ }^{5}$ Baker, R. Controlled Release of Biologically Active Agents. New York: Wiley, p. 279, 1987.

${ }^{6}$ Baldwin, S. P., and W. M. Saltzman. Materials for protein delivery in tissue engineering. Adv. Drug Del. Rev. 33:71-86, 1998.

${ }^{7}$ Basmanav, F. B., G. T. Kose, and V. Hasirci. Sequential growth factor delivery from complexed microspheres for bone tissue engineering. Biomaterials 29:4195-4204, 2008.

${ }^{8}$ Berchane, N. S., K. H. Carson, A. C. Rice-Ficht, and M. J. Andrews. Effect of mean diameter and polydispersity of PLG microspheres on drug release: experiment and theory. Int. J. Pharm. 337:118-126, 2007.

${ }^{9}$ Berkland, C., M. King, A. Cox, K. Kim, and D. W. Pack. Precise control of PLG microsphere size provides enhanced control of drug release rate. J. Control. Release 82:137-147, 2002.

${ }^{10}$ Bilati, U., E. Allemann, and E. Doelker. Strategic approaches for overcoming peptide and protein instability within biodegradable nano- and microparticles. Eur. J. Pharm. Biopharm. 59:375-388, 2005.

${ }^{11}$ Biondi, M., F. Ungaro, F. Quaglia, and P. A. Netti. Controlled drug delivery in tissue engineering. Adv. Drug Del. Rev. 60:229-242, 2008.
${ }^{12}$ Bobo, R. H., D. W. Laske, A. Akbasak, P. F. Morrison, R. L. Dedrick, and E. H. Oldfield. Convection-enhanced delivery of macromolecules in the brain. Proc. Natl Acad. Sci. USA 91:2076-2080, 1994.

${ }^{13}$ Broersma, S. Rotational diffusion constant of a cylindrical particle. J. Chem. Phys. 32:1626-1631, 1960.

${ }^{14}$ Burdick, J. A., M. Ward, E. Liang, M. J. Young, and R. Langer. Stimulation of neurite outgrowth by neurotrophins delivered from degradable hydrogels. Biomaterials 27:452-459, 2006.

${ }^{15}$ Cao, X., and M. S. Shoichet. Defining the concentration gradient of nerve growth factor for guided neurite outgrowth. Neuroscience 103:831-840, 2001.

${ }^{16}$ Chapanian, R., and B. G. Amsden. Combined and sequential delivery of bioactive VEGF165 and HGF from poly(trimethylene carbonate) based photo-cross-linked elastomers. J. Control. Release 143:53-63, 2010.

${ }^{17}$ Chen, R. R., et al. Integrated approach to designing growth factor delivery systems. FASEB J. 21:3896-3903, 2007.

${ }^{18}$ Chen, Q.-Z., et al. An elastomeric patch derived from poly(glycerol sebacate) for delivery of embryonic stem cells to the heart. Biomaterials 31:3885-3893, 2010.

${ }^{19}$ Chew, S. Y., J. Wen, E. K. F. Yim, and K. W. Leong. Sustained release of proteins from electrospun biodegradable fibers. Biomacromolecules 6:2017-2024, 2005.

${ }^{20}$ Chung, C. A., C. W. Chen, C. P. Chen, and C. S. Tseng. Enhancement of cell growth in tissue-engineering constructs under direct perfusion: modeling and simulation. Biotechnol. Bioeng. 97:1603-1616, 2007.

${ }^{21}$ Cohen, S., T. Yoshioka, M. Lucarelli, L. H. Hwang, and R. Langer. Controlled delivery systems for proteins based on poly(lactic/glycolic acid) microspheres. Pharm. Res. 8:713-720, 1991.

${ }^{22}$ Crank, J. The Mathematics of Diffusion. Oxford: Clarendon Press, p. 414, 1975.

${ }^{23}$ Crank, J., and G. S. Park. Diffusion in Polymers. London: Academic Press, p. 452, 1968.

${ }^{24}$ Cruise, G. M., D. S. Scharp, and J. A. Hubbell. Characterization of permeability and network structure of interfacially photopolymerized poly(ethylene glycol) diacrylate hydrogels. Biomaterials 19:1287-1294, 1998.

${ }^{25}$ Cussler, E. L. Diffusion: Mass Transfer in Fluid Systems. New York: Cambridge University Press, p. 580, 1997.

${ }^{26} \mathrm{De}$ La Torre, J. G., and V. A. Bloomfield. Hydrodynamics of macromolecular complexes. II. Rotation. Biopolymers 16:1765-1778, 1977.

${ }^{27}$ Douglas, T., et al. Influence of collagen-fibril-based coatings containing decorin and biglycan on osteoblast behavior. J. Biomed. Mater. Res. A 84A:805-816, 2008.

${ }^{28}$ Duncanson, W. J., M. A. Figa, K. Hallock, S. Zalipsky, J. A. Hamilton, and J. Y. Wong. Targeted binding of PLA microparticles with lipid-PEG-tethered ligands. Biomaterials 28:4991-4999, 2007.

${ }^{29}$ Elcin, Y. M., V. Dixit, and T. Gitnick (eds.). Extensive In Vivo Angiogenesis Following Controlled Release of Human Vascular Endothelial Cell Growth Factor: Implications for Tissue Engineering and Wound Healing. Oxford: Blackwell Science Inc., 2001.

${ }^{30}$ Emerich DF, D. F., et al. (eds.). Biocompatibility of Poly(Dl-Lactide-co-Glycolide) Microspheres Implanted into the Brain. Elmsford, NY: Cognizant Communication Corp., 1999.

${ }^{31}$ Fahmy, T. M., R. M. Samstein, C. C. Harness, and W. M. Saltzman. Surface modification of biodegradable 
polyesters with fatty acid conjugates for improved drug targeting. Biomaterials 26:5727-5736, 2005.

${ }^{32}$ Faisant, N., J. Siepmann, and J. P. Benoit. PLGA-based microparticles: elucidation of mechanisms and a new, simple mathematical model quantifying drug release. Eur. J. Pharm. Sci. 15:355-366, 2002.

${ }^{33}$ Freeman, I., and S. Cohen. The influence of the sequential delivery of angiogenic factors from affinity-binding alginate scaffolds on vascularization. Biomaterials 30:21222131, 2009

${ }^{34}$ Freiberg, S., and X. X. Zhu. Polymer microspheres for controlled drug release. Int. J. Pharm. 282:1-18, 2004.

${ }^{35}$ Guelcher, S. A., and J. O. Hollinger. An Introduction to Biomaterials. Boca Raton, FL: Taylor \& Francis, p. 55, 2006.

${ }^{36}$ Hamidi, M., A. Azadi, and P. Rafiei. Hydrogel nanoparticles in drug delivery. Adv. Drug Del. Rev. 60:16381649,2008

${ }^{37}$ Harris, L. D., B. S. Kim, and D. J. Mooney. Open pore biodegradable matrices formed with gas foaming. J. Biomed. Mater. Res. A 42:396-402, 1998.

${ }^{38}$ Huang, X., and C. S. Brazel. Analysis of burst release of proxyphylline from poly (vinyl alcohol) hydrogels. Chem. Eng. Commun. 190:519-532, 2003.

${ }^{39}$ Jaklenec, A., A. Hinckfuss, B. Bilgen, D. M. Ciombor, R. Aaron, and E. Mathiowitz. Sequential release of bioactive IGF-1 and TGF-beta(1) from PLGA microspherebased scaffolds. Biomaterials 29:1518-1525, 2008.

${ }^{40}$ Jay, S. M., and W. M. Saltzman. Controlled delivery of VEGF via modulation of alginate microparticle ionic crosslinking. J. Control. Release 134:26-34, 2009.

${ }^{41}$ Jeffery, H., S. S. Davis, and D. T. Ohagan. The preparation and characterization of poly(lactide-co-glycolide) microparticles. 2. The entrapment of a model protein using a (water-in-oil)-in-water emulsion solvent evaporation technique. Pharm. Res. 10:362-368, 1993.

${ }^{42}$ Jeon, O., S. J. Song, S.-W. Kang, A. J. Putnam, and B.-S. Kim. Enhancement of ectopic bone formation by bone morphogenetic protein-2 released from a heparin-conjugated poly (1-lactic-co-glycolic acid) scaffold. Biomaterials 28:2763-2771, 2007.

${ }^{43}$ Jiang, G., B. H. Woo, F. Kang, J. Singh, and P. P. DeLuca. Assessment of protein release kinetics, stability and protein polymer interaction of lysozyme encapsulated poly(D, L-lactide-co-glycolide) microspheres. J. Control. Release 79:137-145, 2002.

${ }^{44}$ Kendall, W. F., M. D. Darrabie, H. M. El-Shewy, and E. C. Opara (eds.). Effect of Alginate Composition and Purity on Alginate Microspheres. London: Taylor \& Francis Ltd., 2004.

${ }^{45}$ Klose, D., F. Siepmann, K. Elkharraz, and J. Siepmann. Plga-based drug delivery systems: Importance of the type of drug and device geometry. Int. J. Pharm. 354:95-103, 2008.

${ }^{46}$ Kretlow, J. D., and A. G. Mikos. From material to tissue: biomaterial development, scaffold fabrication, and tissue engineering. AlChE J. 54:3048-3067, 2008.

${ }^{47}$ Leach, J. B., and C. E. Schmidt. Characterization of protein release from photocrosslinkable hyaluronic acidpolyethylene glycol hydrogel tissue engineering scaffolds. Biomaterials 26:125-135, 2005.

${ }^{48}$ Lee, M., B. M. Wu, M. Stelzner, H. M. Reichardt, and J. C. Y. Dunn. Intestinal smooth muscle cell maintenance by basic fibroblast growth factor. Tissue Eng. A 14:13951402, 2008.
${ }^{49}$ Lin, C.-C., and A. T. Metters. Hydrogels in controlled release formulations: network design and mathematical modeling. Adv. Drug Del. Rev. 58:1379-1408, 2006.

${ }^{50}$ Marentette, J. M., and A. E. Grosser. Modeling of the kinetics of drug release from a binary system. J. Pharm. Sci. 81:318-320, 1992.

${ }^{51}$ Matricardi, P., C. Di Meo, T. Coviello, and F. Alhaique. Recent advances and perspectives on coated alginate microspheres for modified drug delivery. Expert Opin. Drug Del. 5:417-425, 2008.

${ }^{52}$ McNeill, M. E., and N. B. Graham. Properties controlling the diffusion and release of water-soluble solutes from poly(ethylene oxide) hydrogels. 2. Dispersion in an initially dry slab. J. Biomater. Sci. Polym. Ed. 5:111-130, 1993.

${ }^{53}$ Mooney, D. J., D. F. Baldwin, N. P. Suh, L. P. Vacanti, and R. Langer. Novel approach to fabricate porous sponges of poly(d, 1-lactic-co-glycolic acid) without the use of organic solvents. Biomaterials 17: 1417-1422, 1996.

${ }^{54}$ Motlagh, D., J. Yang, K. Y. Lui, A. R. Webb, and G. A. Ameer. Hemocompatibility evaluation of poly(glycerol-sebacate) in vitro for vascular tissue engineering. Biomaterials 27:4315-4324, 2006.

${ }^{55}$ Nauman, J. V., P. G. Campbell, F. Lanni, and J. L. Anderson. Diffusion of insulin-like growth factor-1 and ribonuclease through fibrin gels. Biophys. J. 92:4444-4450, 2007.

${ }^{56}$ Nijst, C. L. E., et al. Synthesis and characterization of photocurable elastomers from poly(glycerol-co-sebacate). Biomacromolecules 8:3067-3073, 2007.

${ }^{57}$ Ohagan, D. T., H. Jeffery, and S. S. Davis. The preparation and characterization of poly(lactide-co-glycolide) microparticles. 3. Microparticle/polymer degradation rates and the in vitro release of a model protein. Int. J. Pharm. 103:37-45, 1994.

${ }^{58}$ Patel, M. P., P. Pavlovic, F. J. Hughes, G. N. King, A. Cruchley, and M. Braden. Release of recombinant human bone morphogenetic protein-2 from heterocyclic methacrylate polymer systems. Biomaterials 22:2081-2086, 2001.

${ }^{59}$ Pecora, R. Dynamic light scattering measurement of nanometer particles in liquids. J. Nanopart. Res. 2:123$131,2000$.

${ }^{60}$ Peppas, N. A. Analysis of Fickian and non-Fickian drug release from polymers. Pharm. Acta Helv. 60:110-111, 1985.

${ }^{61}$ Peppas, N. A., Y. Huang, M. Torres-Lugo, J. H. Ward, and J. Zhang. Physicochemical foundations and structural design of hydrogels in medicine and biology. Ann. Rev. Biomed. Eng. 2:9-29, 2000.

${ }^{62}$ Peret, B. J., and W. L. Murphy. Controllable soluble protein concentration gradients in hydrogel networks. Adv. Funct. Mater. 18:3410-3417, 2008.

${ }^{63}$ Perrin, F. The Brownian movement of an ellipsoid-the dielectric dispersion of ellipsoidal molecules. Journal De Physique Et Le Radium 5:497-511, 1934.

${ }^{64}$ Pratt, A. B., F. E. Weber, H. G. Schmoekel, R. Muller, and J. A. Hubbell. Synthetic extracellular matrices for in situ tissue engineering. Biotechnol. Bioeng. 86:27-36, 2004.

${ }^{65}$ Radomsky, M. L., K. J. Whaley, R. A. Cone, and W. M. Saltzman. Macromolecules released from polymers: diffusion into unstirred fluids. Biomaterials 11:619-624, 1990.

${ }^{66}$ Raman, C., C. Berkland, K. Kim, and D. W. Pack. Modeling small-molecule release from PLG microspheres: 
effects of polymer degradation and nonuniform drug distribution. J. Control. Release 103:149-158, 2005.

${ }^{67}$ Rasmussen, M. R., T. Snabe, and L. H. Pedersen. Numerical modelling of insulin and amyloglucosidase release from swelling Ca-alginate beads. J. Control. Release 91:395-405, 2003.

${ }^{68}$ Ribeiro, A. J., C. Silva, D. Ferreira, and F. Veiga. Chitosan-reinforced alginate microspheres obtained through the emulsification/internal gelation technique. Eur. J. Pharm. Sci. 25:31-40, 2005.

${ }^{69}$ Richard, W. B. Membrane Technology and Applications. West Sussex: Wiley, p. 490, 2004.

${ }^{70}$ Richardson, T. P., M. C. Peters, A. B. Ennett, and D. J. Mooney. Polymeric system for dual growth factor delivery. Nat. Biotechnol. 19:1029-1034, 2001.

${ }^{71}$ Ritger, P. L., and N. A. Peppas. A simple equation for description of solute release. I. Fickian and non-Fickian release from non-swellable devices in the form of slabs, spheres, cylinders or discs. J. Control. Release 5:23-36, 1987.

${ }^{72}$ Rosenblatt, J., W. Rhee, and D. Wallace. The effect of collagen fiber size distribution on the release rate of proteins from collagen matrices by diffusion. J. Control. Release 9:195-203, 1989.

${ }^{73}$ Rothstein, S. N., W. J. Federspiel, and S. R. Little. A unified mathematical model for the prediction of controlled release from surface and bulk eroding polymer matrices. Biomaterials 30:1657-1664, 2009.

${ }^{74}$ Rubinstein, M., and R. H. Colby. Polymer Physics. Oxford: Oxford University Press, p. 311, 2003

${ }^{75}$ Saito, N., et al. Local bone formation by injection of recombinant human bone morphogenetic protein-2 contained in polymer carriers. Bone 32:381-386, 2003.

${ }^{76}$ Sakiyama-Elbert, S. E., and J. A. Hubbell. Controlled release of nerve growth factor from a heparin-containing fibrin-based cell ingrowth matrix. J. Control. Release 69:149-158, 2000.

${ }^{77}$ Saltzman, W. M. Drug Delivery: Engineering Principles for Drug Therapy. New York: Oxford University Press, p. $372,2001$.

${ }^{78}$ Saltzman, W. M., and R. Langer. Transport rates of proteins in porous materials with known microgeometry. Biophys. J. 55:163-171, 1989.

${ }^{79}$ Shi, X. T., Y. J. Wang, L. Ren, Y. H. Gong, and D. A. Wang. Enhancing alendronate release from a novel PLGA/hydroxyapatite microspheric system for bone repairing applications. Pharm. Res. 26:422-430, 2009.

${ }^{80}$ Siepmann, J., N. Faisant, and J. P. Benoit. A new mathematical model quantifying drug release from bioerodible microparticles using Monte Carlo simulations. Pharm. Res. 19:1885-1893, 2002.

${ }^{81}$ Siepmann, J., and A. Göpferich. Mathematical modeling of bioerodible, polymeric drug delivery systems. Adv. Drug Del. Rev. 48:229-247, 2001.

${ }^{82}$ Siepmann, J., and N. A. Peppas. Modeling of drug release from delivery systems based on hydroxypropyl methylcellulose (HPMC). Adv. Drug Del. Rev. 48:139-157, 2001.

${ }^{83}$ Sill, T. J., and H. A. von Recum. Electrospinning: applications in drug delivery and tissue engineering. Biomaterials 29:1989-2006, 2008.

${ }^{84}$ Silva, G. A., P. Ducheyne, and R. L. Reis. Materials in particulate form for tissue engineering. 1. Basic concepts. J. Tissue Eng. Regen. Med. 1:4-24, 2007.

${ }^{85}$ Simmons, C. A., E. Alsberg, S. Hsiong, W. J. Kim, and D. J. Mooney. Dual growth factor delivery and controlled scaffold degradation enhance in vivo bone formation by transplanted bone marrow stromal cells. Bone 35:562-569, 2004.

${ }^{86}$ Sinha, V. R., and A. Trehan. Biodegradable microspheres for protein delivery. J. Control. Release 90:261-280, 2003.

${ }^{87}$ Sokolsky-Papkov, M., K. Agashi, A. Olaye, K. Shakesheff, and A. J. Domb. Polymer carriers for drug delivery in tissue engineering. Adv. Drug Del. Rev. 59:187-206, 2007.

${ }^{88}$ Soletti, L., et al. A bilayered elastomeric scaffold for tissue engineering of small diameter vascular grafts. Acta Biomater. 6:110-122, 2010

${ }^{89}$ Stroh, M., W. R. Zipfel, R. M. Williams, S. C. Ma, W. W. Webb, and W. M. Saltzman. Multiphoton microscopy guides neurotrophin modification with poly(ethylene glycol) to enhance interstitial diffusion. Nat. Mater. 3:489-494, 2004.

${ }^{90}$ Stroh, M., W. R. Zipfel, R. M. Williams, W. W. Webb, and W. M. Saltzman. Diffusion of nerve growth factor in rat striatum as determined by multiphoton microscopy. Biophys. J. 85:581-588, 2003.

${ }^{91}$ Sundback, C. A., et al. Biocompatibility analysis of poly(glycerol sebacate) as a nerve guide material. Biomaterials 26:5454-5464, 2005.

${ }^{92}$ Taluja, A., Y. S. Youn, and Y. H. Bae. Novel approaches in microparticulate PLGA delivery systems encapsulating proteins. J. Mater. Chem. 17:4002-4014, 2007.

${ }^{93}$ Thorne, R. G., S. Hrabetova, and C. Nicholson. Diffusion of epidermal growth factor in rat brain extracellular space measured by integrative optical imaging. J. Neurophysiol. 92:3471-3481, 2004

${ }^{94}$ Tobias, I. S., H. Lee, G. C. Engelmayr, Jr., D. Macaya, C. J. Bettinger, and M. J. Cima. Zero-order controlled release of ciprofloxacin- $\mathrm{HCl}$ from a reservoir-based, bioresorbable and elastomeric device. J. Control. Release (in press, corrected proof).

${ }^{95}$ van de Weert, M., W. E. Hennink, and W. Jiskoot. Protein instability in poly(lactic-co-glycolic acid) microparticles. Pharm. Res. 17:1159-1167, 2000.

${ }^{96}$ van Laarhoven, J. A. H., M. A. B. Kruft, and H. Vromans. In vitro release properties of etonogestrel and ethinyl estradiol from a contraceptive vaginal ring. Int. J. Pharm. 232:163-173, 2002

${ }^{97}$ Walters, R. R., J. F. Graham, R. M. Moore, and D. J. Anderson. Protein diffusion-coefficient measurements by laminar-flow analysis - method and applications. Anal. Biochem. 140:190-195, 1984.

${ }^{98}$ Wang, Y., G. A. Ameer, B. J. Sheppard, and R. Langer. A tough biodegradable elastomer. Nat. Biotechnol. 20: 602-606, 2002.

${ }^{99}$ Wei, H. J., et al. Gelatin microspheres encapsulated with a nonpeptide angiogenic agent, ginsenoside Rg1, for intramyocardial injection in a rat model with infarcted myocardium. J. Control. Release 120:27-34, 2007.

${ }^{100}$ Wischke, C., and S. P. Schwendeman. Principles of encapsulating hydrophobic drugs in PLA/PLGA microparticles. Int. J. Pharm. 364:298-327, 2008.

${ }^{101} \mathrm{Wu}$, Y. C., S. Y. Shaw, H. R. Lin, T. M. Lee, and C. Y. Yang. Bone tissue engineering evaluation based on rat calvaria stromal cells cultured on modified PLGA scaffolds. Biomaterials 27:896-904, 2006.

${ }^{102}$ Yamamoto, M., Y. Ikada, and Y. Tabata. Controlled release of growth factors based on biodegradation of gelatin hydrogel. J. Biomater. Sci. Polym. Ed. 12:77-88, 2001. 
${ }^{103}$ Zhang, Y. Z., X. Wang, Y. Feng, J. Li, C. T. Lim, and $\mathrm{S}$. Ramakrishna. Coaxial electrospinning of (fluorescein isothiocyanate-conjugated bovine serum albumin)-encapsulated poly( $\varepsilon$-caprolactone) nanofibers for sustained release. Biomacromolecules 7:1049-1057, 2006.
${ }^{104}$ Zhong, Y. H., and R. V. Bellamkonda. Biomaterials for the central nervous system. J. R. Soc. Interface 5:957-975, 2008.

${ }^{105}$ Zhu, G. Z., S. R. Mallery, and S. P. Schwendeman. Stabilization of proteins encapsulated in injectable poly (lactide-co-glycolide). Nat. Biotechnol. 18:52-57, 2000. 